\title{
Rotation and Anisotropic Losses of Mass and Angular Momentum
}

\author{
André Maeder \\ Geneva Observatory, CH - 1290 Sauverny, Switzerland
}

\begin{abstract}
The expressions of the radiative flux at the surface of a non-uniformly rotating star are revised and this leads to a small extra-term in the von Zeipel theorem. The Eddington factor needs also to be carefully defined in a rotating star, as well as the critical break-up velocity. This leads us to reconsider the so-called $\Omega$-limit. The most massive stars reach the $\Omega$ and $\Gamma$ limits almost simultaneously.

We also examine the latitudinal dependence of the mass loss rates $\dot{M}(\vartheta)$ in rotating stars and find two main effects: 1$)$ the " $g_{\text {eff }}$ " effect which enhances the polar ejection; 2) the "opacity effect" (or " $\kappa$-effect"), which favours equatorial ejection. In $\mathrm{O}$-stars, the $g_{\text {eff }}$ effect is expected to largely dominate. In B- and later type stars the opacity effect should favour equatorial ejection and the formation of equatorial rings. Possible relations with $\eta$ Carinae and the inner and outer rings of SN $1987 \mathrm{~A}$ are mentioned. Opacity peaks produce some extrema in $\dot{M}(\vartheta)$ and this may also lead to the formation non-equatorial symmetrical rings.

Anisotropic stellar winds remove selectively the angular momentum. For example, winds passing through polar caps in $\mathrm{O}$-stars remove very little angular momentum, an excess of angular momentum is retained and rapidly redistributed by horizontal turbulence. These excesses may lead some Wolf-Rayet stars, those resulting directly from $\mathrm{O}$-stars, to be fast spinning objects, while we predict that the WR-stars which have passed through the red supergiant phase will have lower rotation velocities on the average. We also show how anisotropic ejection can be treated in numerical models by properly modifying the outer boundary conditions for the transport of angular momentum.
\end{abstract}

\section{Introduction}

Rotation has effects in the deep stellar interiors and also at the stellar surfaces. The main effects in the interior results from the transport of chemical elements and of angular momentum by shear turbulence and meridional circulation (cf. Zahn, 1992; Maeder, 1997; Maeder and Zahn, 1998). Here, in accordance with the thema of the meeting, we shall examine some of the interesting problems concerning the coupling of mass loss and rotation, which are important for stellar evolution. We may mention in particular the distribution of the radiative flux at the stellar surface, the correct expressions of the Eddington factor $\Gamma$ and of the critical velocity in rotating stars, the latitudinal dependence of the mass loss rates $\dot{M}(\vartheta)$, the loss and gain of angular momentum in the remaining star as a result of the anistropies in the mass loss rates, etc. 


\section{The flux and the Eddington factor $\Gamma$}

On the surface of a rotating star, the gravity, $T_{\text {eff }}$ and the flux are not constant. The flux is generally given by the von Zeipel theorem (von Zeipel, 1924), which states that a given colatitude $\vartheta$

$$
\mathbf{F}=-\frac{L(P)}{4 \pi G M_{\star}(P)} \mathbf{g}_{\text {eff }}
$$

with

$$
M_{\star}=M\left(1-\frac{\Omega^{2}}{2 \pi G \rho_{m}}\right)
$$

where $\rho_{m}$ is the average density inside the considered surface level. This allows us to know the local $T_{\text {eff }}$

$$
T_{\text {eff }}(\vartheta) \sim g_{\text {eff }}^{1 / 4}(\vartheta)
$$

The von Zeipel theorem usually applies to constant or cylindrical rotation. However, stars are likely to have more complicated rotation law, such as the "shellular rotation" proposed by Zahn (1992). Such a law of the form $\Omega=\Omega(r)$ results from the strong horizontal turbulence which rapidly damps the horizontal differences of the rotation. A more general expression of the von Zeipel theorem has been obtained (cf. Maeder, 1999), it is

$$
\mathbf{F}=\frac{-L(P)}{4 \pi G M_{\star}(P)} \mathbf{g e f f}_{\mathbf{e f}}(\mathbf{1}+\zeta(\vartheta))
$$

with

$$
\begin{gathered}
\zeta(\vartheta)=\frac{H_{T}}{\delta} \frac{d \Theta}{d r} P_{2}(\cos \vartheta) \\
\Theta=\frac{1}{3} \frac{r^{2}}{\bar{g}} \frac{d \Omega^{2}}{d r}
\end{gathered}
$$

The term $\zeta$ is of the order of a few $10^{-2}$, with $P_{2}(\cos (\vartheta))=1$ at the pole and -0.5 at the equator. Thus, the term $\zeta(\vartheta)$ contributes to enhance the radiative flux at the pole and to decrease it at the equator.

The Eddington factor $\Gamma$ is usually taken as

$$
\Gamma=\frac{L \kappa}{4 \pi c G M} .
$$

It needs to be redefined more precisely in the case of a rotating star, since both the flux and the gravity are varying over the stellar surface. We consider a local $\Gamma(\vartheta)$ taken as the ratio of the stellar flux to the local limiting flux, i.e. 
André Maeder

$$
\Gamma(\vartheta)=\frac{F(\vartheta)}{F_{\lim }(\vartheta)}
$$

The limiting flux is obtained from the condition

$$
\mathbf{g}_{\text {tot }}=\mathbf{g}_{\text {eff }}+\mathbf{g}_{\text {rad }}=\mathbf{g}_{\mathbf{g r a v}}+\mathbf{g}_{\mathrm{rot}}+\mathbf{g}_{\mathbf{r a d}}
$$

which shows that the limiting geff and grad have the same direction, which introduces a major simplfication. Thus, the limiting flux is

$$
\mathbf{F}_{\text {lim }}=\frac{-c}{\kappa} \mathbf{g}_{\text {eff }}
$$

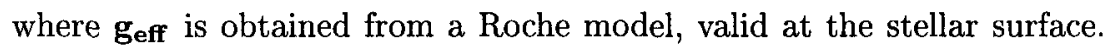
Thus, we finally have for the Eddington factor in a rotating star

$$
\Gamma(\vartheta)=\frac{\kappa(\vartheta) L(P)}{4 \pi c G M_{\star}(P)}[1+\zeta(\vartheta)]
$$

We note three differences between expressions (7) and (11). 1) The $\zeta-$ term favours a higher $\Gamma$ at the pole. 2) The opacity $\kappa(\vartheta)$ applies only locally, which means that if $\kappa$ grows for lower T, $\Gamma$ is larger at the equator. 3) $\Gamma$ depends also on rotation.

\section{The $\Omega$ and $\Gamma$ limits}

These results are very useful in relation with the concept of an " $\Omega$-limit" recently introduced by Langer (1997) and Heger and Langer (1998). The central idea is that if one has, for the critical break-up velocity

$$
v_{\mathrm{crit}}^{2}=\frac{G M}{R}(1-\Gamma)
$$

the value of $v_{\text {crit }}^{2}$ would then tend towards zero for a star approaching the Eddington limit. Thus, Langer's conclusion was that for any non-zero rotation a star would reach its critical rotation velocity (the " $\Omega$-limit") before the formal Eddington limit.

Indeed, one has, for the total gravity taking into account von Zeipel's theorem,

$$
\mathbf{g}_{\text {tot }}=\mathbf{g}_{\text {eff }}\left[1-\frac{\kappa(\vartheta) L(1+\zeta(\vartheta))}{4 \pi c G M\left(1-\frac{\Omega^{2}}{2 \pi G \rho_{m}}\right)}\right]
$$

The break-up velocity occurs when rotation is such that $\mathbf{g}_{\text {tot }}=0$ at the equator. We see that expression (13) has formally two roots, one given by $\mathbf{g}_{\text {eff }}=0$ and the other obtained by nulling the bracket term in (13). The condition $\mathbf{g}_{\text {eff }}=0$ at the equator gives the usual expression 


$$
v_{\text {crit }}^{2}=\Omega^{2} r_{e b}^{2}=\frac{G M}{r_{e b}}
$$

where $r_{e b}$ is the equatorial radius at break-up velocity. Thus we see that expression (12) is not correct since the current expression $v_{\text {crit }}^{2}$ does not contain any $\Gamma$. The reason for this is simply that close to break-up the effective gravity goes down to zero, and so does the radiative flux according to von Zeipel's theorem. Solving the surface equation, we find for the second root of (13)

$$
v_{\text {crit }}^{2}=3.3256 \frac{G M}{r_{e b}}\left[1-\frac{\kappa(\vartheta) L(P)}{4 \pi c G M}\left(1+\zeta\left(\frac{\pi}{2}\right)\right)\right]
$$

which is generally bigger than the first root given by (13), except when $\frac{\kappa(\vartheta) L(P)}{4 \pi c G M}(1+\zeta(\vartheta))$ is bigger than 0.6993 . When the rotation velocity grows, the root which is met first determines the physically significant zero of (13) and thus the critical velocity. We can conclude that (14) is the general expression for the critical velocity, except if $\frac{\kappa(\vartheta) L}{4 \pi c G M}(1+\zeta(\vartheta))$ happens to become larger than 0.6993 , a situation which, if it does occur, will only concern stars very close to the Eddington limit. Thus, the $\Omega$ and $\Gamma$ limits are met almost simultaneously.

\section{Mass loss in rotating stars}

\subsection{Latitudinal variations}

According to the radiative wind theory (cf. Pauldrach et al. 1986; Kudritzki et al. 1989; Puls et al. 1996) the mass loss rates are essentially scaling like

$$
\dot{M} \sim(k \alpha)^{1 / \alpha}\left(\frac{1-\alpha}{\alpha}\right)^{\frac{1-\alpha}{\alpha}} F(\vartheta)^{1 / \alpha} g_{\text {tot }}^{1-\frac{1}{\alpha}}(\vartheta)
$$

where $k$ and $\alpha$ are the force multiplier parameters. $F(\vartheta)$ is the local flux as given by the revised von Zeipel theorem. The values of $k$ and $\alpha$ are changing with $\mathrm{T}_{\text {eff }}$ (cf. Pauldrach et al. 1986); for $\mathrm{T}_{\text {eff }}=50000,40000,30000$ and $20000 \mathrm{~K}$ one has respectively $k=0.124,0.124,0.17,0.32$, and $\alpha=0.64,0.64$, $0.59,0.565$. Then, taking into account the expression for the flux with the revised von Zeipel theorem, as well as $g_{\text {tot }}$, we obtain for the mass flux,

$$
\dot{M}(\vartheta) \sim(k \alpha)^{\frac{1}{\alpha}}\left(\frac{1-\alpha}{\alpha}\right)^{\frac{1-\alpha}{\alpha}}\left[\frac{L(P)}{4 \pi G M_{\star}(P)}\right]^{\frac{1}{\alpha}} \frac{g_{\mathrm{eff}}(1+\zeta(\vartheta))^{\frac{1}{\alpha}}}{(1-\Gamma(\vartheta))^{\frac{1}{\alpha}-1}}
$$

This relation expresses the dependence of the mass loss rates on colatitude. If $\alpha$ and $k$ are constant in latitude (as normally expected in O-stars), we see that $\dot{M}(\vartheta)$ mainly depends on $g_{\text {eff }}$ (cf. also Owocki et al. 1996, 1998). This means that in a rotating hot star the mass loss rates by unit surface 
are much larger over the polar caps than at the equator. The terms $\zeta(\vartheta)$ and $\Gamma(\vartheta)$ in (17) slightly reinforce the polar mass loss.

As mentioned above $k$ and $\alpha$ vary with $\mathrm{T}_{\text {eff }}$. This means that over the surface of a rotating star $k$ and $\alpha$ also vary. The term $(k \alpha)^{\frac{1}{2}}\left(\frac{1-\alpha}{\alpha}\right)^{\frac{1-\alpha}{\alpha}}$ increases by a factor of three from $\mathrm{T}_{\text {eff }}=50000 \mathrm{~K}$ to $20000 \mathrm{~K}$. The term in brackets in (17) is also larger for lower $\alpha$-values. The term containing $\Gamma$ increases with the value of $\Gamma$, this growth being much faster in case of lower $\alpha$-values. On the whole the following picture emerges: 1 . In hot, rotating stars the mass flux is higher at the poles and lower at the equator (the respective surface areas must of course be accounted for in numerical models). Let us call this the "geff-effect" in rotating stars. 2. This behaviour is also present near the $\Omega \Gamma$-limit where the mass flux is strongly increased as shown by (17). 3. In B and later type stars the enhanced polar ejection is progressively compensated by effects of larger line opacities (higher $k$ and lower $\alpha$ ), which favour progressively larger mass flux in the cooler equatorial regions. We shall call this the " $\kappa$-effect" in rotating stars. 4 . For B and later type stars near the $\Omega \Gamma$-limit the mass flux is strongly enhanced, particularly in the equatorial regions. The wind density also has important latitudinal variations (cf. Maeder, 1999).

A so-called bistability of stellar winds has been found by Lamers (1997) in non-rotating stars: near $\mathrm{T}_{\text {eff }}=20000 \mathrm{~K}$ and also close to $10000 \mathrm{~K}$, large and rather abrupt changes of the force multiplier parameters $k$ and $\alpha$ modify the relations between $v_{\infty}$ and $v_{\text {esc }}$ and the mass loss rates. These important changes of $k$ and $\alpha$ should also occur in B- and A-type rotating stars, as a result of the decrease of $\mathrm{T}_{\text {eff }}$ between the pole and the equator.

\subsection{The case of $\eta$ Carinae and of the rings in SN $1987 \mathrm{~A}$}

The HST pictures of $\eta$ Carinae show two broad polar ejections and an equatorial skirt (cf. Ebbets et al. 1997; Davidson 1997). $\eta$ Carinae is clearly a hot star close to the $\Omega \Gamma$-limit. Among the various explanations possible for the observed geometry of the ejections from $\eta$ Carinae we might point out the possibility that polar ejections result from the "geff-effect" in (17) while the equatorial skirt is more likely to stem from the " $\kappa$-effect".

The complex structure around SN 1987 A consists of a bright elliptical inner ring and of two outer rings moved away from the central ring (cf. Crotts et al. 1989; Burrows et al. 1995). Currently the two outer rings are interpreted as real rings and not as rings due to the limb brightening of an hour glass shell (cf. Burrows et al. 1995). Their location and CNO composition suggest (cf. Panagia et al. 1996) that they were ejected at an earlier stage of evolution than the inner ring, perhaps when the SN progenitor was a blue supergiant. The bright inner ring is generally associated to the red supergiant stage in view of its composition, location and the timescales involved. We notice that an equatorial ring could be consistent with the " $\kappa$-effect" in cool stars while symmetrical outer rings would better correspond with peaks in the function $\dot{M}(\vartheta)$ due for example to some opacity peaks. 
We must really wonder about the possibility of sharp extrema of the functions $\dot{M}(\vartheta)$ given by (17). This appears as a likely possibility. Indeed, if for some $\vartheta$, the $\mathrm{T}_{\text {eff }}$ is such that there is a peak or a discontinuity in the opacity, $\dot{M}(\vartheta)$ will also show corresponding features at this colatitude. The net result will be the formation of rings. As a matter of fact, the very strong variations of $\alpha$ (cf. Lamers et al. 1995), which changes abruptly at $\mathrm{T}_{\text {eff }}=20000$ and $10000 \mathrm{~K}$, may produce steep enhancements in $\dot{M}(\vartheta)$ and in the wind density and this may lead to the formation of symmetrical rings in nebulae. Future numerical models will tell us what are the features in the observed anisotropic nebulae which can be accounted for by the above rotational effects.

\section{Change of angular momentum as a result of anisotropic mass loss}

The anisotropic mass loss demonstrated above may have major consequences for stellar evolution. For example, polar ejection, as in O-type stars, removes very little angular momentum, and in particular much less than if the mass loss rates would be the same over the stellar surface. This implies that the angular momentum not embarked by polar winds remains as an excess $\mathcal{L}_{\text {excess }}$ in the outermost layers. This excess is rapidly redistributed within these layers by strong horizontal turbulence which operates on short timescales. Conversely equatorial mass loss will remove more angular momentum.

The above considerations imply that we must carefully rediscuss the surface boundary conditions applicable to the equation expressing the conservation of angular momentum in a rotating star. In particular, we find that the change of the angular momentum of the last shell between $r$ and $R$ is (Maeder, 1999)

$$
\frac{\partial}{\partial t}\left[\bar{\Omega} \int_{r}^{R} \rho r^{4} d r\right]=-\frac{1}{5} \rho r^{4} \bar{\Omega}[U(r)-5 \dot{r}]+\mathcal{L}_{\text {excess }}
$$

The second member expresses the transport by the meridional circulation (term with $U(r)$ ), the effect of contraction or expansion (term with $\dot{r}$ and the effect of the anisotropic mass loss.

Let us now express the excess of angular momentum $\mathcal{L}_{\text {excess }}$ applied to the last remaining shell as a result of the upper inhomogeneous mass removal. This excess is the difference between the angular momentum $\mathcal{L}(\Omega)$ of the last shell and the angular momentum $\mathcal{L}_{\text {anis }}(\Omega)$ anisotropically removed by mass loss. One has

$$
\mathcal{L}_{\text {excess }}(\Omega)=\mathcal{L}(\Omega)-\mathcal{L}_{\text {anis }}(\Omega)=J(\Omega) \Omega\left[1-\frac{J_{\text {anis }}(\Omega)}{J(\Omega)}\right]
$$


$J(\Omega)$ is the moment of inertia of a shell at the surface of a rotating star of angular velocity $\Omega$ while $J_{\text {anis }}(\Omega)$ is the moment of inertia of the mass which is ejected by stellar winds. $\mathcal{L}_{\text {excess }}$ will be positive for a polar ejection an negative for an equatorial one. The application of this new boundary condition will modify the angular momentum during the evolution' of mass losing stars.Thus, it is likely that some WR stars, which after the $\mathrm{O}$-phase have always stayed on the blue side of the HR diagram are fast rotators, since polar ejection is likely to have been dominant. At the opposite, WR stars resulting from an evolution through the red-supergiant phase, where equatorial mass loss dominates, may show lower rotational velocities on the average.

\section{References}

Burrows C.J., Krist J., Hester J.J. et al. 1995, ApJ 452, 680

Crotts A.P.S., Kunkel W.E., McCarthy P.J. 1989, ApJ 347, L61

Davidson K. 1997, Bull. American Astron. Soc. 188, 50.04.

Ebbets D.C., Walborn N.R., Parker J. 1997, ApJ 489, L161

Heger A., Langer N. 1996, A\&A 315, 421

Kudritzki R.P., Pauldrach A., Puls J., Abbott D.C. 1989, A\&A 219, 205

Lamers H.J.G.L.M. 1997, in Luminous Blue Variables: Massive Stars in Transition, ed. A. Nota, H. Lamers, ASP Conf. Ser. 120, 76

Lamers H.J.G.L.M., Snow T.P., Lindholm D.M. 1995, ApJ 455, 269

Langer N. 1997, in Luminous Blue Variables: Massive Stars in Transition, ed. A. Nota, H. Lamers, ASP Conf. Ser. 120, p. 83

Maeder A. 1997, A\&A 321, 134 (paper II)

Maeder A. 1999, A\&A in press (paper IV)

Maeder A., Zahn J.P. 1998, A\&A 334, 1000 (paper III)

Owocki S.P., Cranmer S.R., Gayley K.G. 1996, ApJ 472, L115

Owocki S.P., Gayley K.G., Cranmer S.R. 1998, in Boulder-Munich II: Properties of Hot, Luminous Stars, ASP Conf. Ser. 131, p. 237

Panagia N., Scuderi S., Gilmozzi R., Challis P.M., Garnavich P.M., Kirshner R.P. 1996, ApJ 459, L17

Pauldrach A., Puls J., Kudritzki R.P. 1986, A\&A 164, 86

Puls J., Kudritzki R.P., Herrero A. et al. 1996, A\&A 305, 171

von Zeipel H. 1924, MNRAS 84, 665

Zahn J.P. 1992, A\&A 265, 115

\section{Discussion}

J. Puls: In the model of $\zeta$ Pup $(\Gamma=0.6)$ Peter Petrenz presented in his poster paper, we included both the effects of gravity darkening and a latitudedependent $\Gamma$, as well as a latitude-dependent $\alpha$. As a result, the mass loss was still larger over the poles, since $\alpha$ remained roughly constant. The major 
change was in the velocity field, which was faster at the equator due to the lower $\Gamma$ (higher $v_{\mathrm{esc}}$ ).

A. Maeder: I agree that for O-type stars not at break-up velocity, gravity darkening is insufficient to reduce the equatorial $\mathrm{T}_{\text {eff }}$ enough to make a lower $\alpha$. Thus polar ejection dominates. For B stars, however, the reduction of $\alpha$ (i.e., the increase of opacity) at the equator may overcome the gravity darkening effect, so as to produce equatorial ejection. Concerning the velocity field, I would like to remind you of my remark that in general $v_{\text {esc }}$ does not depend on $\Gamma$.

H. Lamers: Concerning the question of mass loss increasing toward the pole or the equator, you should not forget that observations tell us that there are disks around hot stars. These observations are, to name just a few: polarisation; the relation between polarisation and $v_{\text {rot }}$ for Be stars; the doublepeaked line profiles at low velocity of low ionisation lines; and the X-ray variability of Be-X-ray binaries. Although it may be possible to explain some of these effects by different models (e.g., polarisation by polar outflows) it is in my opinion impossible to explain all of these together by polar flows. For instance, polar flows might give double-peaked profiles, but that will be in high ionisation species (hot polar caps) and high wind velocity (high $v_{\text {esc }} \rightarrow$ high $v_{\infty}$ ).

F.-J. Zickgraf: Could your models also explain $\mathrm{N}$ enhancement on the main sequence for a $20 \mathrm{M}_{\odot}$ star? This would be of importance for the $\mathrm{B}[\mathrm{e}]$ star $\mathrm{R} 4$ for which we have found an $\mathrm{N}$ overabundance. We had interpreted this with a post-red supergiant evolutionary stage. In connection with the results of Norbert Langer, the $\mathrm{N}$ overabundance at an early stage in the evolution could indicate that the first arrival at the $\Omega$-limit is important for the origin of $\mathrm{B}[\mathrm{e}]$ stars.

A. Maeder: The first models show that $20 \mathrm{M}_{\odot}$ stars on the main sequence may undergo $\mathrm{N}$-enhancement due to rotational mixing. I do not know which is the minimum mass for which this is possible. This may be an interesting point of comparison between models and observations.

S. Owocki: A general comment: I think we must be careful not to think that evidence for a "disk" must mean an equatorially enhanced stellar wind. A disk might not be outflowing at all, but in a stationary Keplerian orbit. Such Keplerian disks would likely have an origin quite independent of the wind.

R. Schulte-Ladbeck: I have to contradict what Henny said, at least from the point of view of spectropolarimetry of massive stars - it is not possible to tell from the data whether the material is in the equator or over the poles of the star. I do think the evidence for disks is strong only in the case of Be stars, owing to the existence of a large sample of stars observed with polarimetry, for which we can analyse the statistical probability of the distribution 
of projection angles on the plane of the sky. A possibility to distinguish an equatorial disk from a distribution of matter over the poles exists in principle (with polarimetry) in the case of binaries. Analysing a polarimetric time series can tell you whether material is located mainly in the binary (and hence equatorial) plane or over the pole (1st harmonic vs. 2nd harmonic). Finally, the behaviour of the polarisation across emission lines (see my paper on $\mathrm{EZ}$ $\mathrm{CMa}$ ) might also become a useful tool to detect disks: unlike polar plumes, they rotate.

H. Henrichs: How do your evolutionary tracks depend upon the inclination of the star? If one wants to derive evolutionary masses from the observed luminosity and effective temperature, the inclination angle is apparently an important factor.

A. Maeder: I am pleased to say that we know the changes in $M_{V}$ and $(B-V)$ produced by rotation for various orientation angles rather well (cf., Maeder \& Peytremann 1970, A\&A 7, 120). As an example, at extreme rotation for pole-on stars, the excess brightness obtained by integration of the local fluxes over the visible part of the star is about $0.5 \mathrm{mag}$ in $\mathrm{M}_{\mathrm{V}}$, the change in $(\mathrm{B}-\mathrm{V})$ is close to zero; for equator-on stars, the decrease of brightness is about 0.15 mag for a $5 \mathrm{M}_{\odot}$ and $0.4 \mathrm{mag}$ for a $2 \mathrm{M}_{\odot}$ star. There is, however, an important reddening of the colour for equator-on stars. Other values can be obtained from the tables.

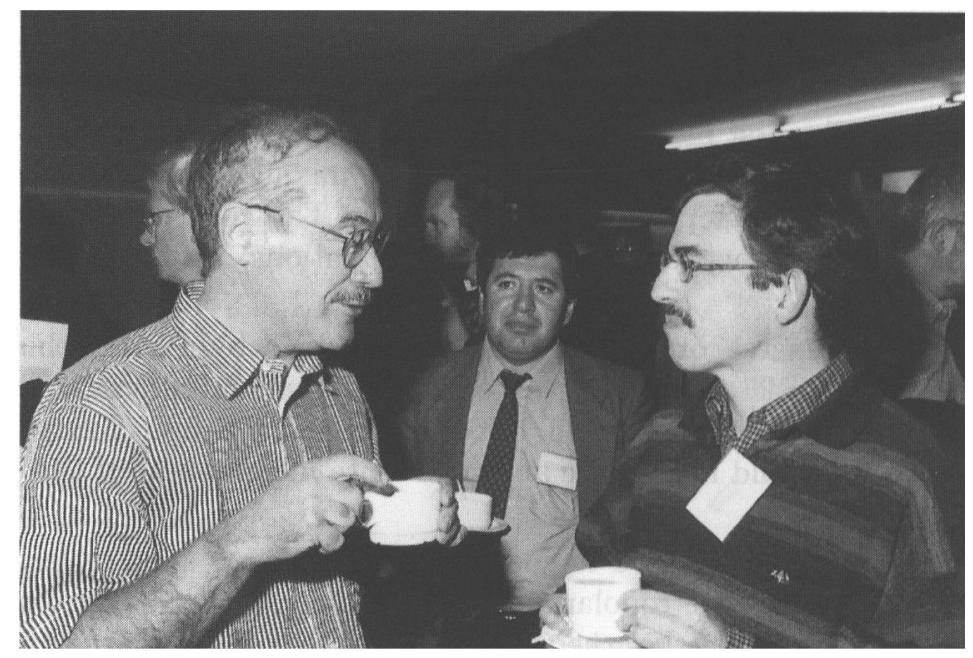

André Maeder and Rens Waters (Valeri Hambaryan in the background) 